\title{
Adaptive Multiscale Noise Control Enhanced Stochastic Resonance Method Based on Modified EEMD with Its Application in Bearing Fault Diagnosis
}

\author{
Jimeng $\mathrm{Li}^{1}$ and Jinfeng Zhang ${ }^{2}$ \\ ${ }^{1}$ College of Electrical Engineering, Yanshan University, Qinhuangdao 066004, China \\ ${ }^{2}$ College of Liren, Yanshan University, Qinhuangdao 066004, China \\ Correspondence should be addressed to Jimeng Li; xjtuljm@163.com
}

Received 30 June 2016; Accepted 28 September 2016

Academic Editor: Ganging Song

Copyright (C) $2016 \mathrm{~J}$. Li and J. Zhang. This is an open access article distributed under the Creative Commons Attribution License, which permits unrestricted use, distribution, and reproduction in any medium, provided the original work is properly cited.

\begin{abstract}
The structure of mechanical equipment becomes increasingly complex, and tough environments under which it works often make bearings and gears subject to failure. However, effective extraction of useful feature information submerged in strong noise that is indicative of structural defects has remained a major challenge. Therefore, an adaptive multiscale noise control enhanced stochastic resonance (SR) method based on modified ensemble empirical mode decomposition (EEMD) for mechanical fault diagnosis is proposed in the paper. According to the oscillation characteristics of signal itself, the algorithm of modified EEMD can adaptively decompose the fault signals into different scales and it reduces the decomposition levels to improve calculation efficiency of the proposed method. Through filter processing with the constructed filters, the orthogonality of adjacent intrinsic mode functions (IMFs) can be improved, which is conducive to enhancing the extraction of weak features from strong noise. The constructed signal obtained by using IMFs is inputted into the SR system, and the noise control parameter of different scales is optimized and selected with the help of the genetic algorithm, thus achieving the enhancement extraction of weak features. Finally, simulation experiments and engineering application of bearing fault diagnosis demonstrate the effectiveness and feasibility of the proposed method.
\end{abstract}

\section{Introduction}

With the fast development of modern technology, the structure of mechanical equipment becomes increasingly complex, and the automation degree becomes more and more high, but tough environments under which it works often make bearings and gears subject to failure. The failure may deteriorate mechanical performance and even lead to fatal breakdowns. Therefore, how to ensure the safe and reliable operation of mechanical equipment and reduce economic losses is increasingly becoming a hot topic concerned by enterprises. The health monitoring and fault diagnosis technology provides an effective solution for the predictive maintenance of mechanical equipment operating state. In view of the inherent link between machine operation and vibration, vibration signals collected from mechanical equipment carry rich information on machine health conditions. Therefore, vibration signal analysis [1-3] has been extensively investigated during the past decades. The emergence of periodic components is often associated with component failures in vibration analysis. However, fault signals collected from mechanical equipment are often corrupted by strong noise coming from other coupled machine components and the working environment, which increases the difficulty in fault identification. Thus, effective extraction of weak features submerged in strong noise that are indicative of structural defects has remained a major challenge. In order to obtain vital feature information from vibration signals, various signal processing techniques, like variational mode decomposition (VMD) $[4,5]$, synchrosqueezing transform (SST) [6, 7], wavelet transform (WT) [8-10], and so forth, have been extensively studied and used in machinery fault diagnosis. Traditionally, noise is always considered an undesirable disturbance, thus these signal processing techniques mainly focus on filtering and denoising the signals to extract fault features. In fact, noise is not just a source of signal contamination, but it also 
represents a kind of signal energy. If the energy generated by noise can be used properly, it is conducive to extracting the feature information from the signals submerged under strong noise. Stochastic resonance (SR) [11] is a kind of typical noise-assisted data processing method. Unlike traditional denoising techniques, $S R$ realizes the detection of weak signal by utilizing noise instead of eliminating noise, and the weak signal features are not weakened but enhanced.

The concept of SR was first introduced in the 1980s, which was applied to describe the periodicity associated with Earth's ice ages in climatology $[12,13]$. SR, as a nonlinear physical phenomenon, emphasizes the synergistic effect between a nonlinear dynamic system, a small parameter signal, and noise. As a special "low-pass filter," SR can transfer noise energy to useful signal with the assistance of nonlinear system, thereby realizing the elimination of noise and enhancement of useful signal features. Consequently, by virtue of the unique advantage of using noise to enhance weak signals, SR has been widely studied in machinery fault diagnosis field $[14,15]$. Qin et al. [16] proposed an adaptive and fast SR method based on dyadic wavelet transform and least square parameters solving algorithm to extract the fault feature of a rotor system, which can increase the noise utilization and does not need to set up the searching range and searching step size of system parameters. Aiming at the problem of detecting the multifrequency signals buried under strong noise, Han et al. [17] proposed a multistable SR method by using wavelet transform and parameter compensation band-pass algorithm, which enhanced the signal amplitude and achieved the effective extraction of bearing fault features. Wang et al. [18] proposed an adaptive multiscale noise tuning SR based on wavelet transform and weighted power kurtosis to diagnose the rolling bearing fault and realized the adaptive selection of control parameters through the artificial fish swarm algorithm. In order to realize the fault diagnosis of planetary gear transmission, a weak fault feature information extraction method based on ensemble empirical mode decomposition (EEMD) and adaptive SR was proposed [19]. Through EEMD, the effective IMFs containing main fault feature information are selected and reconstructed. Then the reconstructed signal is inputted into adaptive SR system, and the weak fault feature information can be extracted from the output signal of SR system. By analyzing the SR phenomenon of a two-dimensional duffing oscillator, Lai and Leng [20] proposed a generalized parameter-adjusted SR model, which can be used for the fault diagnosis of rolling bearing. In addition, many researches have also been conducted on the SR model and SR enhancement methods [21, 22].

The algorithm of multiscale noise tuning provides an effective control strategy for realizing the SR. However, there are some shortcomings in the method of multiscale noise tuning based on wavelet decomposition, such as the selection of optimal wavelet bases, which makes it have some limitations in processing diverse signals in engineering practice. Adaptive signal decomposition methods provide an effective solution for complicated signal analysis and feature extraction, which can adaptively decompose the signal into different scales according to the oscillation characteristics of signal itself, thus avoiding artificial intervention and optimizing the data analysis flow. As a noise-assisted adaptive signal decomposition method, EEMD can eliminate the mode mixing phenomenon in empirical mode decomposition (EMD) through adding white noise to signal, and it decomposes the complicated signal into a set of simple components named intrinsic mode function (IMF) [23-25]. The IMFs represent the natural oscillatory mode embedded in the signal, which are determined by the signal itself, instead of predetermined kernels. Therefore, a new adaptive multiscale noise control enhanced SR method based on modified EEMD for bearing fault diagnosis is studied by using the adaptive decomposition capability of EEMD and the unique advantage of SR using noise to enhance weak signal features. EEMD is used for signal multiscale decomposition, and through improving the EEMD algorithm, the decomposition levels are reduced to increase the computation efficiency of the proposed method. The band-pass filters are constructed in terms of the spectrum distribution characteristics of IMFs, so as to eliminate some aliasing components and improve the orthogonality of adjacent IMFs through filter processing, which is conducive to enhancing and extracting of weak information features to some extent. And then, the signal, used as the input of SR system, is reconstructed with the IMFs obtained by the modified EEMD algorithm, and the noise control parameter of different scales is optimized and selected with the help of the genetic algorithm to achieve the extraction of weak features. Therefore, the proposed method can not only adaptively decompose the signal into different scales but also realize the adaptive selection of noise control parameter of different scales, which is well-suited for enhancement of rotating machine weak fault identification. Experiments and application demonstrate that the proposed method is validated to be effective in detecting the weak fault features in bearing fault diagnosis.

The rest of the paper is arranged as follows. A brief introduction to the theoretical background of SR is provided in Section 2. The method of adaptive multiscale noise control enhanced SR based on modified EEMD is described in detail in Section 3. Some experimental and practical data are applied to verify the effectiveness of the proposed method in Section 4. Finally, conclusions are drawn in Section 5.

\section{SR Basic Theory}

SR describes a phenomenon that the weak signal is enhanced and the noise is weakened through the interaction of the nonlinear system, small parameter signal, and noise, whose dynamic behavior can be represented by the Brownian motion equation of particles. And the overdamped SR equation with a nonlinear bistable model in the presence of periodic force and noise can be written as follows:

$$
\frac{\mathrm{d} x(t)}{\mathrm{d} t}=a x(t)-b x(t)^{3}+A \sin (2 \pi f t+\varphi)+n(t),
$$

where $x(t)$ denotes the system output, parameters $a$ and $b$ are positive real numbers, $A$ and $f$ are the amplitude and frequency of the periodic force, respectively, and $n(t)$ is a Gaussian white noise with zero mean and $D$ variance. The potential function is $U(x, t)=-(a / 2) x(t)^{2}+(b / 4) x(t)^{4}$ with 
two stable points $x= \pm \sqrt{a / b}$ and one critical stable point $x_{0}=$ 0 . The height of potential barrier is $\Delta U=a^{2} / 4 b$. From (1), the system output $x(t)$ is actually the Brownian particle trajectory in the potential function $U(x)$ under the combined action of periodic force and noise. And, the crucial process of using SR to detect weak signal is to adjust system parameters or noise intensity to make the transition rate of the particle caused by noise match the oscillation period of the input signal, thus amplifying the particle movements in single potential well to the transition motions between double potential wells to realize the feature enhancement of input signal.

It is generally known that the theory basis of SR is the adiabatic approximation theory, which requires the amplitude and frequency of periodic signal as well as noise intensity to be smaller than 1 . However, the defective signals caused by components fault of rotating machine have difficulty in satisfying the above requirements in engineering application. Therefore, in order to solve the problem of using SR to detect large parameter signals, several parameter tuning SR methods have been researched based on a normalized scale transformation.

Mathematically, let $y=x \sqrt{b / a}, \tau=a t$, and (1) be written as

$$
\frac{\mathrm{d} y}{\mathrm{~d} \tau}=y-y^{3}+\sqrt{\frac{b}{a^{3}}}\left[A \sin \left(\frac{2 \pi f \tau}{a}+\varphi\right)+n\left(\frac{\tau}{a}\right)\right] .
$$

Equation (2) indicates that the frequency of the periodic signal is normalized to be $1 / a$ times that of original signal through the normalized scale transform, and the normalized frequency of larger parameter signal can satisfy the small parameters requirements of SR for input signal by selecting a corresponding larger parameter $a$. And importantly, according to (1) and the derivation process of (2), the process of realizing SR by adjusting the system parameters is consistent with that of realizing SR by adjusting the input signal strength and noise intensity on the premise of the constant system parameters in nature. Consequently, we can adjust the input signal strength and noise intensity to realize the SR detection of weak signal.

\section{Adaptive Multiscale Noise Control Enhanced SR Method Based on Modified EEMD}

Recent studies demonstrate that the SR effect can be driven by different scales of noise but with different degree [26]. Therefore, according to the influence of noise at different scales on the SR, multiscale noise tuning SR methods based on wavelet transform have been studied in recent years $[27,28]$. However, based on the inner product transform principle, the selection of wavelet bases has important effects on the decomposition results in wavelet transform. When the selected wavelet base is inappropriate, namely, it does not match up with the target signal, the useful signal features may be impaired. Additionally, the width of frequency bands obtained by wavelet decomposition is fixed, which cannot be adjusted adaptively according to the oscillation characteristics of signal itself. Therefore, the existing multiscale noise tuning methods based on wavelet transform still have some shortcomings, which have difficulty in satisfying the demand of the diversity of signals in engineering practice. EMD, as an adaptive signal processing method, has been developed and widely applied in machinery fault diagnosis. Based on the local characteristic time scales of a signal, EMD can adaptively decompose the complicated signal into a set of IMF components. However, it has a major drawback, which is the mode mixing problem. Therefore, EEMD, as an improved method of EMD, is presented to alleviate the mode mixing problem in EMD. The principle of EEMD algorithm is the following: by using the statistical property that Gaussian white noise is uniformly distributed over the whole frequency range, Gaussian white noise is added to a signal, which makes the signal continuous in different scales to alleviate the mode mixing problem in EMD. Finally, the added white noise can be decreased or even completely canceled out through the ensemble mean of enough trials, and the ensemble mean is treated as the true answer. Therefore, according to the oscillation characteristics of signal itself, EEMD algorithm can realize the adaptive multiscale decomposition for input signal, which provides a new control strategy for realizing SR by adjusting multiscale noise. EEMD overcomes the mode mixing problem through enough iterations and trails which is at the cost of increasing the calculation amount. However, in consideration of the requirements of the multiscale noise control SR algorithm in this study, the algorithm of EEMD is modified to simplify the calculation, and a new adaptive multiscale noise control enhanced SR method based on modified EEMD is proposed. By using the oscillation characteristics of signal itself, the modified EEMD algorithm can adaptively decompose the signal into different scales, and by adjusting the noise intensity of different scales the proposed method can achieve the enhanced extraction of weak signal features. The important steps of the proposed method are described in detail below.

3.1. Modified EEMD Algorithm. As mentioned above, EEMD achieves the adaptive partition of frequency band for input signal, and the decomposition level $n$ is not set by manual but depends on the local characteristic time scales of signal itself. Therefore, how to select the appropriate IMF components from the $n$ IMFs obtained by EEMD, namely, that determines the reconstruction scale $L$ to reconstruct the signal used as the input of SR system, is the key of realizing the multiscale noise control SR method based on EEMD.

According to the process of EMD algorithm extracting IMF components, the high-frequency components contained in the signal are extracted first, and the low-frequency components are extracted last. So, the obtained $n$ IMFs are arranged in descending order by the center frequency of each IMF component. And the process of determining the reconstruction scale $L$ is to seek out the $L$ th IMF component containing the target signal from $n$ IMFs, thereby obtaining the first $L$ IMFs which are served as the reconstruction components of multiscale noise control algorithm, and the remainder $n-L$ IMFs below the target signal frequency are abandoned. Aiming at the requirements of the proposed method, EEMD is applied to decompose the signal into 
(1) Initialize: $r_{0}(t) \leftarrow x_{i}(t), j \leftarrow 1$, and $E r r_{j-1} \leftarrow f_{s} / 2$

(2) Extract the $j$ th IMF $c_{i j}(t)$

(a) $h_{j(p-1)}(t) \leftarrow r_{j-1}(t)$, and $p \leftarrow 1$

(b) Extract the local maxima and minima of $h_{j(p-1)}(t)$, and obtain the mean $m_{j(p-1)}(t)$ of upper and lower envelops of $h_{j(p-1)}(t)$ by cubic spline interpolation

(c) $h_{j p}(t) \leftarrow h_{j(p-1)}(t)-m_{j(p-1)}(t)$

(d) If $h_{j p}(t)$ is an IMF then set $c_{i j}(t)=h_{j p}(t)$, else go to step (a) with $p \leftarrow p+1$

(3) Calculate the difference value $E r r_{j}$ between the center frequency $f_{j}$ of $c_{i j}(t)$ and the frequency $f_{0}$ of target signal

(a) Calculate the discrete Fourier transform $C_{i j}(k)$ of $c_{i j}(t)$

(b) Calculate the center frequency $f_{j}$ of $c_{i j}(t)$

$$
f_{j} \longleftarrow \frac{\sum_{k=1}^{N / 2}\left[k\left(f_{s} / N\right)\left|C_{i j}(k)\right|^{2}\right]}{\sum_{k=1}^{N / 2}\left|C_{i j}(k)\right|^{2}}
$$

(c) $\operatorname{Err}_{j} \leftarrow\left|f_{j}-f_{0}\right|$

(4) Determine the terminal condition

If $E r r_{j}>E r r_{j-1}$, the decomposition process is finished, and the obtained IMFs are $c_{i}(t)=\left\{c_{i 1}(t), c_{i 2}(t), \ldots, c_{i j}(t)\right\}$,

$j=1,2, \ldots, L+1$, and the IMF $c_{i L}$ corresponding to the decomposition scale $L$ includes the target signal,

else $r_{j}(t)=r_{j-1}(t)-c_{i j}(t)$. If $r_{j}(t)$ still has least 2 extremum then go to step (2) with $j=j+1$.

Algorithm 1: The algorithm of modified EMD.

different scales, but the algorithm of multiscale noise control does not need all of the IMFs obtained by EEMD but just the first $L$ IMFs. For this reason, the stopping criterion for iteration of EMD algorithm extracting IMF components is modified, and thus a modified EEMD algorithm is presented, which can effectively reduce the decomposition levels of EMD algorithm to improve the overall calculation efficiency of the proposed method in the paper. below:

The modified EEMD algorithm is described in detail

(1) Initialize the number of ensemble Num, the standard deviation of added white noise $\sigma$.

(2) Add a Gaussian white noise with the given standard deviation $\sigma$ to the investigated signal $x(t)$ with the length of $N$ and the sample frequency $f_{s}$,

$$
x_{i}(t)=x(t)+n_{i}(t) \quad i=1,2, \ldots, \text { Num, }
$$

where $n_{i}(t)$ denotes the $i$ th added white noise and $x_{i}(t)$ indicates the noise-added signal of the $i$ th trial.

(3) Decompose the noise-added signal $x_{i}(t)$ with the modified EMD algorithm and determine the decomposition scale $L$ by using the target signal frequency $f_{0}$ and the center frequency $f_{j}$ of each IMF obtained in the decomposition process, thus obtaining $L+1$ IMFs. The concrete decomposition process is described as shown in Algorithm 1.

(4) Repeat steps (2) and (3) again and again, but with different white noise each time, and calculate the ensemble mean $\bar{c}_{j}$ of the Num trials for each IMF:

$$
\bar{c}_{j}=\frac{1}{\operatorname{Num}} \sum_{i=1}^{\mathrm{Num}} c_{i j}(t), \quad j=1,2, \ldots, L+1 .
$$

(5) Conduct the mean $\bar{c}_{j}$ of each of the $L+1$ IMFs as the final IMFs.

It is clear that the $L+1$ IMFs can be obtained through the modified EEMD algorithm, and the $L$ th IMF $\bar{c}_{L}$ of them includes the target signal. Accordingly, the first $L$ IMFs are applied to reconstruct the signal used as the input of SR system. Obviously, the modified EEMD algorithm effectively reduces the computation load and improves the efficiency of the proposed method. And the IMF components below the target signal frequency are abandoned to avoid the influence of low-frequency components on the detection result of SR and improve the detection accuracy of target signal.

3.2. Construction of Filters Based on IMFs. According to the oscillation characteristics of signal itself, EEMD can partition the frequency band adaptively, and the bandwidth of each IMF component depends on the natural oscillation mode embedded in the signal, instead of being determined by human being. However, due to the influence of strong background noise, the obtained IMFs are not strictly orthogonal to each other, as shown in Figure 1. Therefore, based on the spectrum distribution characteristics of $L+1$ IMFs obtained by modified EEMD, the $L+1$ IMFs are employed to construct the corresponding filters to filter the $L$ IMFs, so as to eliminate the spectrum energy leakage and improve the orthogonality of adjacent IMFs.

From Figure 1, it is observed that the frequency spectrum distribution of the first IMF component represents the highpass characteristics and the rest of IMF components show the band-pass characteristics in frequency domain. Accordingly, the corresponding high-pass and band-pass filters can be constructed by using the frequency spectrum distribution characteristics of IMFs. 


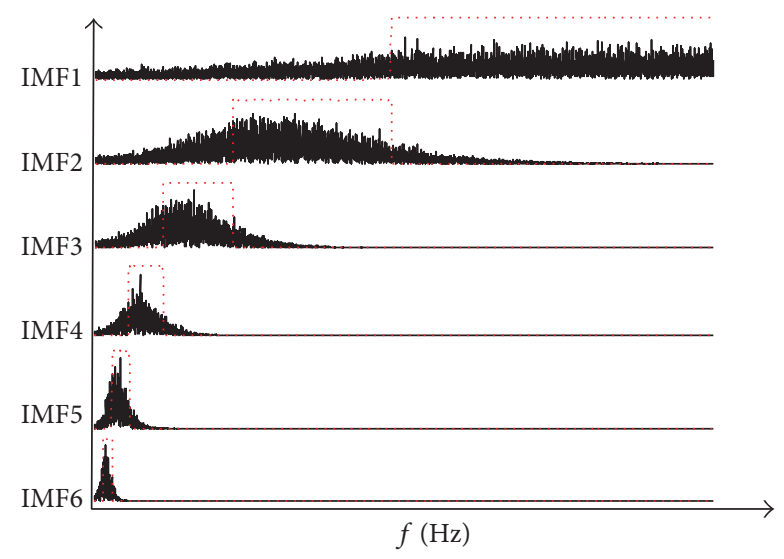

FIGURE 1: Construction sketch of filters based on IMFs.

First, according to the frequency spectrum characteristics of the first IMF component, the parameters of high-pass filter are designed as follows:

$$
\begin{aligned}
& f_{1 p}=\frac{\left(f_{1}+f_{2}\right)}{2}, \\
& f_{1 s}=f_{1 p}-50,
\end{aligned}
$$

where $f_{1 p}$ is the cutoff frequency of passband and $f_{1 s}$ is the cutoff frequency of stopband. $f_{1}$ and $f_{2}$ are the center frequencies of the first and second IMF components, respectively.

Similarly, using the frequency spectrum characteristics of each $\operatorname{IMF}_{j}(j=2,3, \ldots, L)$, the parameters of band-pass filter corresponding to each IMF component are constructed as follows:

$$
\begin{aligned}
& f_{j p 1}=\frac{\left(f_{j}+f_{j+1}\right)}{2}, \\
& f_{j s 1}=f_{j p 1}-10, \\
& f_{j p 2}=\frac{\left(f_{j}+f_{j-1}\right)}{2}, \\
& f_{j s 2}=f_{j p 2}+10,
\end{aligned}
$$

$$
j=2,3, \ldots, L,
$$

where $f_{j s 1}$ and $f_{j s 2}$ are the stopband lower limit frequency and upper limit frequency, respectively, and $f_{j p 1}$ and $f_{j p 2}$ are the passband lower limit frequency and upper limit frequency, respectively. And the stopband attenuation and passband attenuation of the constructed filters are $60 \mathrm{~dB}$ and $0.1 \mathrm{~dB}$, respectively. $f_{j}$ is the center frequency of the $j$ th IMF component. It is worth noting that the parameters of filters in (5) and (6) are the reference values, which can be appropriately adjusted according to the specific requirements on the premise of ensuring the orthogonality of adjacent filters as much as possible. In Figure 1, the amplitudefrequency curves of the constructed filters corresponding to the IMF components are denoted by the red dotted line. Obviously, these constructed filters preserve good orthogonality at boundaries, and the filter processing for the $L$ IMFs with the constructed filters can effectively improve the orthogonality of adjacent IMFs.

3.3. Multiscale Reconstruction with Noise Intensity of Different Scales. The $L$ IMFs obtained by the modified EEMD algorithm are filtered with the constructed filters and their corresponding filtered results $R_{j}(j=1,2, \ldots, L)$ are adjusted with a controllable parameter $\alpha$ to realize the noise control of different scales and then employed to reconstruct the input signal. The reconstruction formula is as follows:

$$
\tilde{x}=\sum_{j=1}^{L}\left(2^{\alpha+j} \cdot R_{j}\right) \quad j=1,2, \ldots, L,
$$

where $\tilde{x}$ is the reconstructed signal, $\alpha$ is a controllable parameter, which denotes the noise intensity of different scales, and $R_{j}$ is the filtered $j$ th IMF component.

3.4. Algorithm Flow of Adaptive Multiscale Noise Control Enhanced SR Based on Modified EEMD. The algorithm procedure of adaptive multiscale noise control enhanced SR based on modified EEMD is illustrated in Figure 2. The concrete steps of this algorithm are as follows.

(1) Vibration Signal Preprocessing. The vibration signals collected from bearings are demodulated by the Hilbert envelop analysis, and the obtained envelope signals are used as the input of modified EEMD algorithm.

(2) Parameter Initialization. Set the SR system parameters $a=$ 1 and $b=1$, the searching range of noise intensity, the initial parameters of genetic algorithm, and the standard deviation of added white noise and the ensemble number of modified EEMD algorithm.

(3) Multiscale Decomposition and Filter Processing. The envelop signal obtained by preprocessing is adaptively decomposed into $L+1$ IMFs by using modified EEMD algorithm and then by using the frequency spectrum distribution characteristics of $L+1$ IMFs to construct the corresponding filters, which are applied to filter the $L$ IMFs.

(4) Adaptive Multiscale Noise Control SR. Equation (7) is applied to reconstruct the input signal with the filtered $L$ IMFs, and then the reconstructed signal $\tilde{x}$ is entered into the rescaling SR system. The genetic algorithm is employed to adaptively select and optimize noise intensity $\alpha$, whose fitness function is constructed by using the signal-to-noise ratio (SNR) of resonance output. And based on the maximum of resonance output SNR, the optimal noise intensity $\alpha$ of different scales can be obtained, thus realizing the resonance detection of input signal. The calculation formula of SNR is as follows:

$$
\mathrm{SNR}=10 \log 10\left(\frac{A_{d}}{A_{n}}\right),
$$

where $A_{d}$ is the amplitude value corresponding to the driving signal frequency and $A_{n}$ is the sum of all the amplitude values except $A_{d}$ in the amplitude spectrum. 


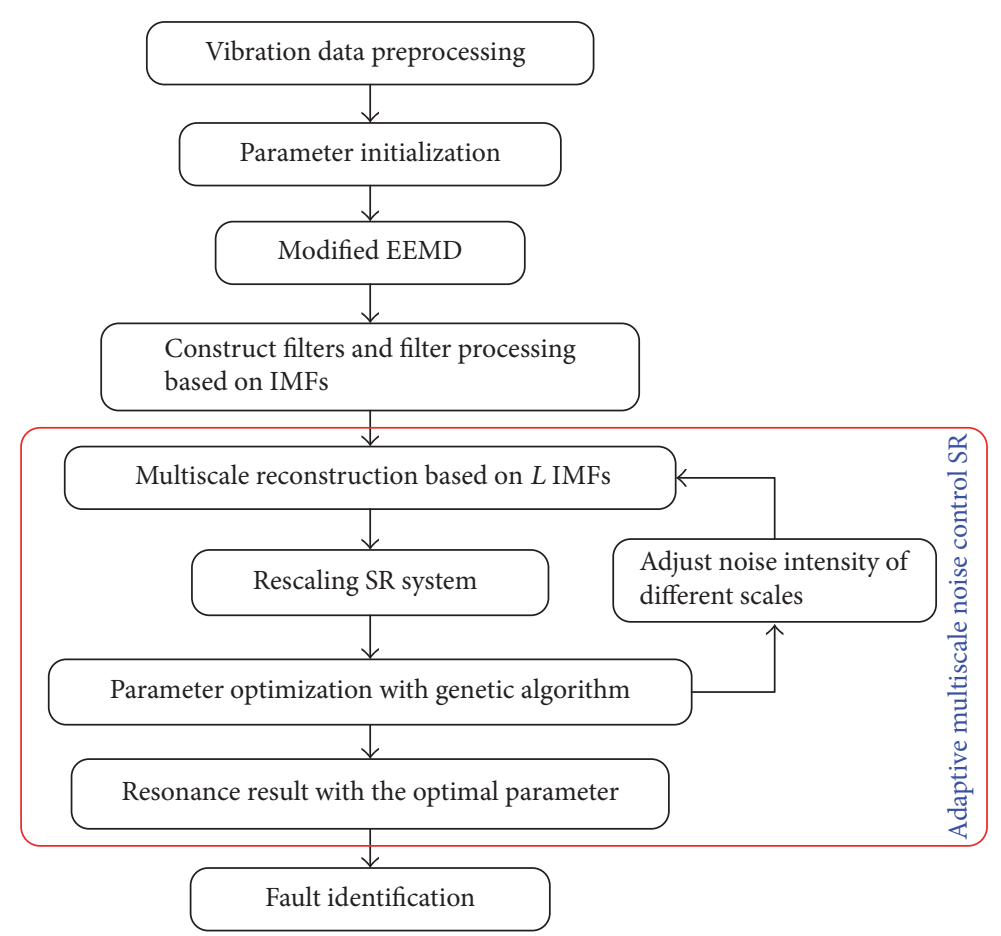

FIGURE 2: Flow diagram of adaptive multiscale noise control enhanced SR algorithm based on modified EEMD.

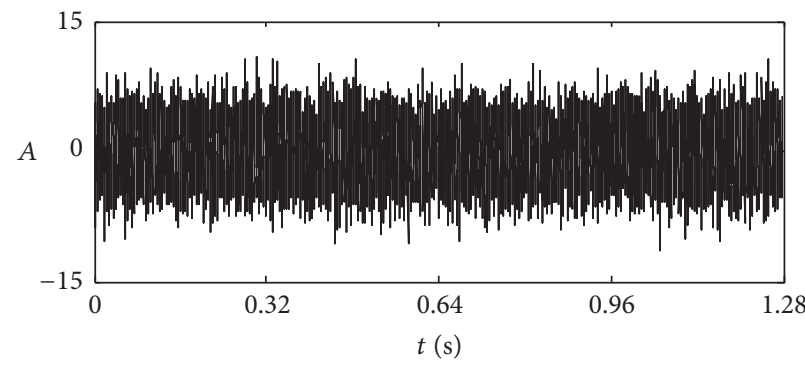

(a)

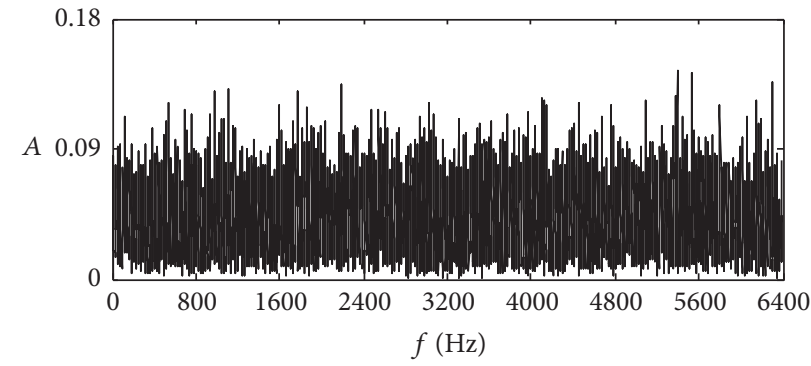

(b)

FIGURE 3: Simulation signal: (a) the original waveform; (b) the frequency spectrum.

(5) Fault Identification. Combined with the mechanical equipment parameters information, the running status of bearing can be identified and diagnosed with SR detection results.

\section{Experiment and Engineering Application}

4.1. Numerical Simulation. In this subsection, the proposed method is applied to extract weak periodic signal from strong noise and analyze the important role of filter processing in the proposed algorithm. The simulation signal is defined as $x(t)=0.09 \sin (100 \pi t)+n(t)$, and $n(t)$ is Gaussian white noise with zero mean and standard deviation of 3 . The sample frequency is $f_{s}=12800 \mathrm{~Hz}$, and the length of data is $N=16384$. The time waveform of simulation signal $x(t)$ is shown in Figure 3(a), and Figure 3(b) is the corresponding frequency spectrum. It can be found that the periodic component is completely submerged by strong noise and cannot be distinguished.

The simulation signal is processed by the proposed method in this study. The searching range of noise intensity $\alpha$ is $[0.1,10]$, the standard deviation of added white noise is 0.08 , and the ensemble number is 50 . The genetic algorithms parameters settings are as follows: the number of initial population is 50 , the maximum number of generations is 25 , the precision of the variables is $1 e-8$, and so forth. Based on the maximum of output SNR of SR system, the optimal noise intensity obtained by genetic algorithm is $\alpha=5.6$, and the corresponding detection results are displayed in Figure 4. Figure 4(a) indicates the time waveform of resonance output of SR system, and the noise is reduced significantly. Furthermore, the spectrum peak at $50 \mathrm{~Hz}$ is very prominent in the frequency spectrum, as shown in Figure 4(b), thus realizing the effective extraction of weak periodic component from the strong background noise. 


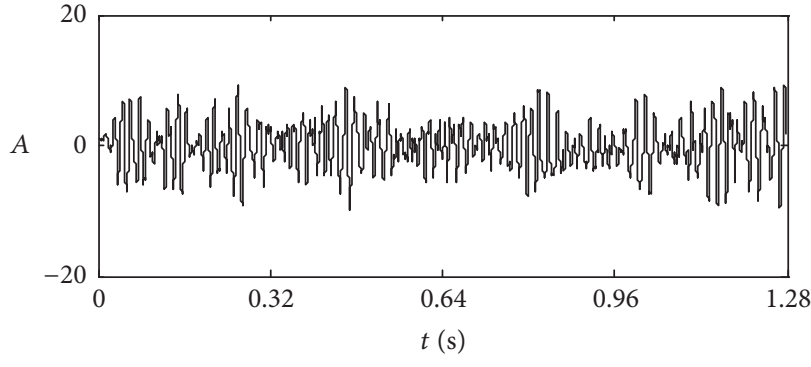

(a)

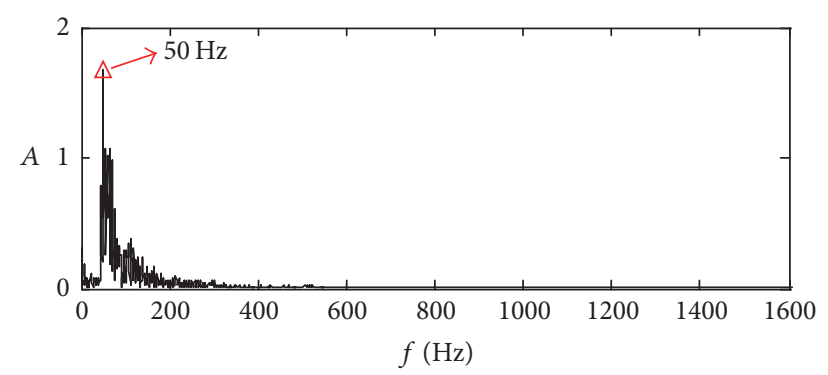

(b)

FIGURE 4: Detection result: (a) the output waveform of SR system; (b) the frequency spectrum of resonance output.

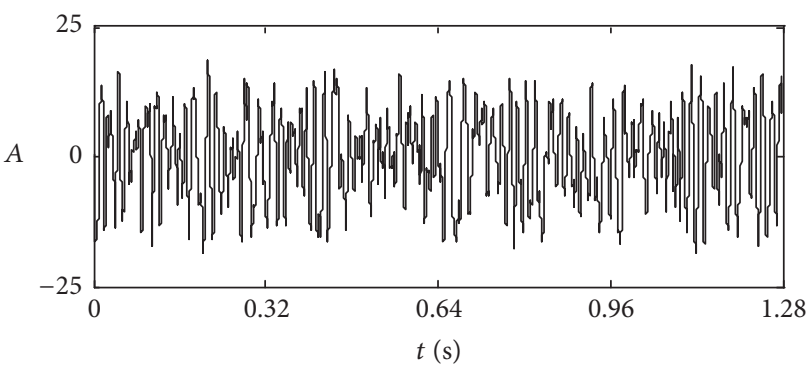

(a)

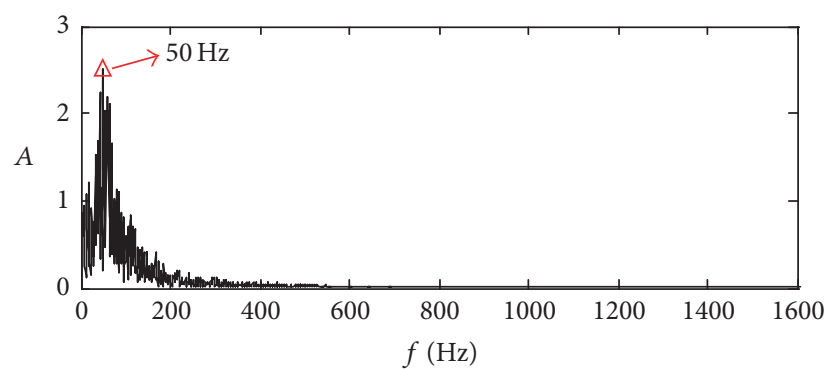

(b)

FIGURE 5: Detection result: (a) the output waveform of SR system; (b) the frequency spectrum of resonance output.

In order to illustrate the important role of the filtering processing in the proposed method, a comparison result is provided in Figure 5. In the algorithm flow of the proposed method shown in Figure 2, the part of construction of filters and filtering processing is removed; namely, the obtained $L$ IMFs are used directly to reconstruct the input signal with (7). Figure 5 displays the time waveform of detection result and its corresponding frequency spectrum. Although the periodic component submerged in strong noise can be extracted, there still exists more interference nearby the spectrum peak at $50 \mathrm{~Hz}$, as shown in Figure 5(b), which is less obvious than that of Figure 4. In consequence, the comparison results indicate that through constructing the corresponding filters and filter processing of the $L$ IMFs, the proposed method effectively eliminates the interference of useless components and achieves the enhancement and extraction of feature information in the weak signal detection.

4.2. Experiment Verification. To verify the effectiveness and efficiency of the proposed method in engineering practice, some vibration data collected from bearings are analyzed with the proposed method shown in Figure 2.

(a) Rolling Element Fault Detection of Bearing. In view of the structure features of rolling bearing, when damage occurs in rolling elements, the useful information features that are indicative of structural defects are weak and submerged in strong noise, which makes it difficult to extract. Therefore, fault diagnosis for rolling elements of bearings has remained a major challenge. In this paper, vibration data of bearings that are from the Case Western Reserve University Bearing
Data Center website [29] are analyzed by the proposed method. Fan end bearing was used as the analysis object, and the rolling element fault was generated by electrodischarge machining with fault diameter of $0.178 \mathrm{~mm}$ and depth of $0.279 \mathrm{~mm}$. The type of fan end bearing was $6203-2 \mathrm{RS}$ JEM SKF, the motor speed was $1777 \mathrm{r} / \mathrm{min}$, and the sample frequency was $12000 \mathrm{~Hz}$. According to the bearing parameters, the fault characteristic frequency of rolling element is $118.09 \mathrm{~Hz}$, and the length of data is 16384 .

Figure 6 displays the rolling element fault signal and its corresponding frequency spectrum. It is observed that the time waveform is disorderly, and the frequency components are abundant and useful features relevant to rolling element fault can hardly be identified in the frequency spectrum. In the envelop spectrum, as shown in Figure 6(c), the frequency component $117.9 \mathrm{~Hz}$ that is consistent with the fault characteristic frequency of rolling element can be found, but it is weak and not obvious, making it hard to identify the fault efficiently.

The proposed method in the paper is applied to process the vibration signal caused by rolling element fault. The algorithm parameters are the same as in the previous section except for the reaching range of noise intensity $\alpha$, which is set to $[0.1,20]$. The detection results obtained by the proposed method are displayed in Figure 7. The processing results indicate that the high-frequency signal energy is concentrated to target signal through SR system, and some useless low-frequency interferences are eliminated, which makes the spectrum peak at $117.9 \mathrm{~Hz}$ more outstanding in the frequency spectrum. Accordingly, the weak fault feature of rolling element of bearing is enhanced and extracted 


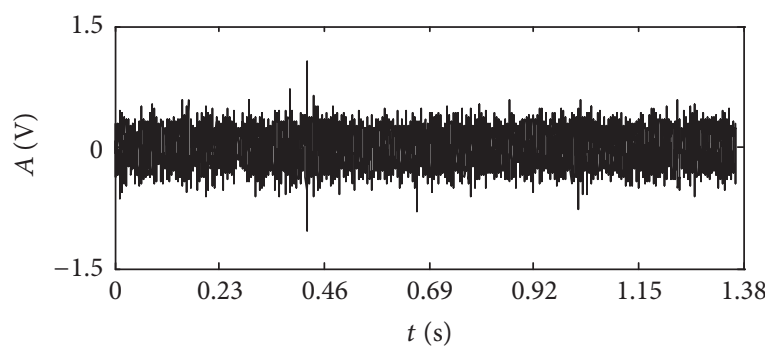

(a)

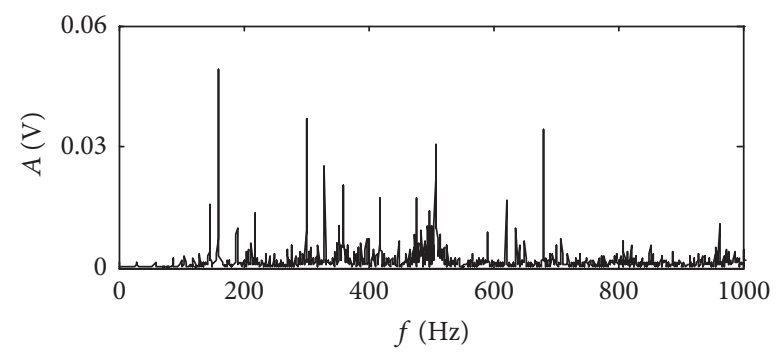

(b)

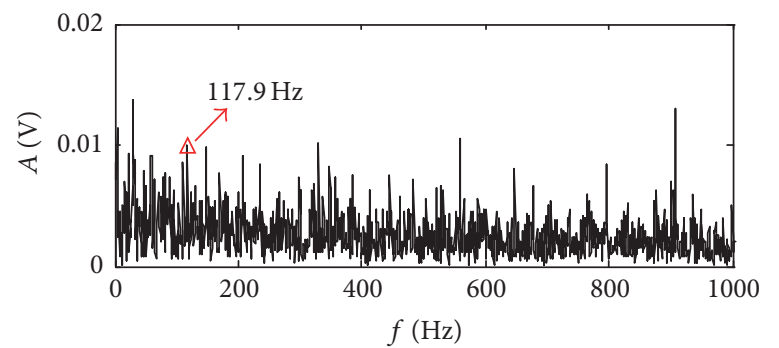

(c)

FIGURE 6: Vibration signal of bearing: (a) the time waveform; (b) the frequency spectrum; (c) the envelop spectrum.

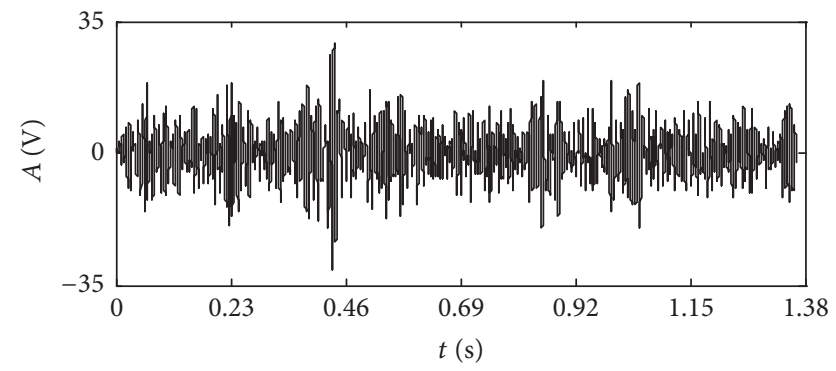

(a)

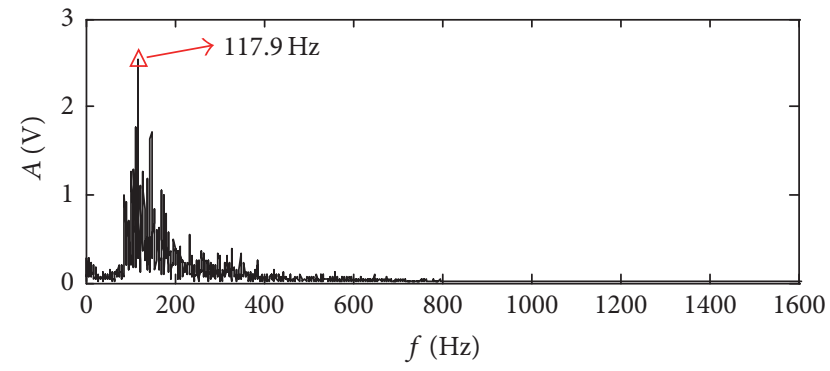

(b)

FIGURE 7: Detection results of the proposed method: (a) the output waveform of SR system; (b) the frequency spectrum.

effectively by using the proposed method to improve the accuracy of fault diagnosis.

Besides, the analyzed results of EEMD method with the parameters of the number of ensembles being 50 and the standard deviation of the added white noise being 0.08 are illustrated in Figure 8. Obviously, the EEMD method fails to provide the effective decomposition results, and it is difficult to find the characteristic frequency $117.9 \mathrm{~Hz}$ in each IMF and its corresponding envelop spectrum. Therefore, the detection results of the proposed method are superior to those of the envelop spectrum analysis of EEMD.

(b) Inner Race Fault Detection of Rolling Bearing. The vibration data of bearing fault were collected from a fault motor of Spectra Quest Inc.'s mechanical fault simulator with acceleration sensors, as shown in Figure 9. In the experiment, the employed bearing with inner race defect was the deep groove ball bearing with the type of $6203 \mathrm{SKF}$, and the motor rotating speed was $1433 \mathrm{r} / \mathrm{min}$. The sample frequency was $6400 \mathrm{~Hz}$, and the length of data was 16384 . Combining the information of bearing parameters and its corresponding rotating frequency, it can be derived that the fault characteristic frequency of inner race of bearing is $117.52 \mathrm{~Hz}$.

Figure 10(a) shows the time waveform of bearing signal, and it is difficult to identify the feature information relevant to inner race fault. In Figure 10(b), the frequency components are abundant, and apart from the rotating frequency, the prominent spectrum peak fails to be found at the fault characteristic frequency of bearing inner race. And in the Hilbert envelop spectrum, as Figure 10(c) shows, besides the rotating frequency of $23.83 \mathrm{~Hz}$, the inconspicuous spectrum peak at $117.2 \mathrm{~Hz}$ can be found, which is approximately consistent with the fault characteristic frequency of bearing inner race. However, due to the influence of interference frequency components, the feature information of inner race fault is still not easy to be identified to detect faults.

The proposed method is applied to analyze the vibration signal. The algorithm parameters are the same as in the previous section except for the reaching range of noise intensity $\alpha$, which is set to $[0.1,20]$. And the corresponding detection results obtained by the proposed method are displayed in 

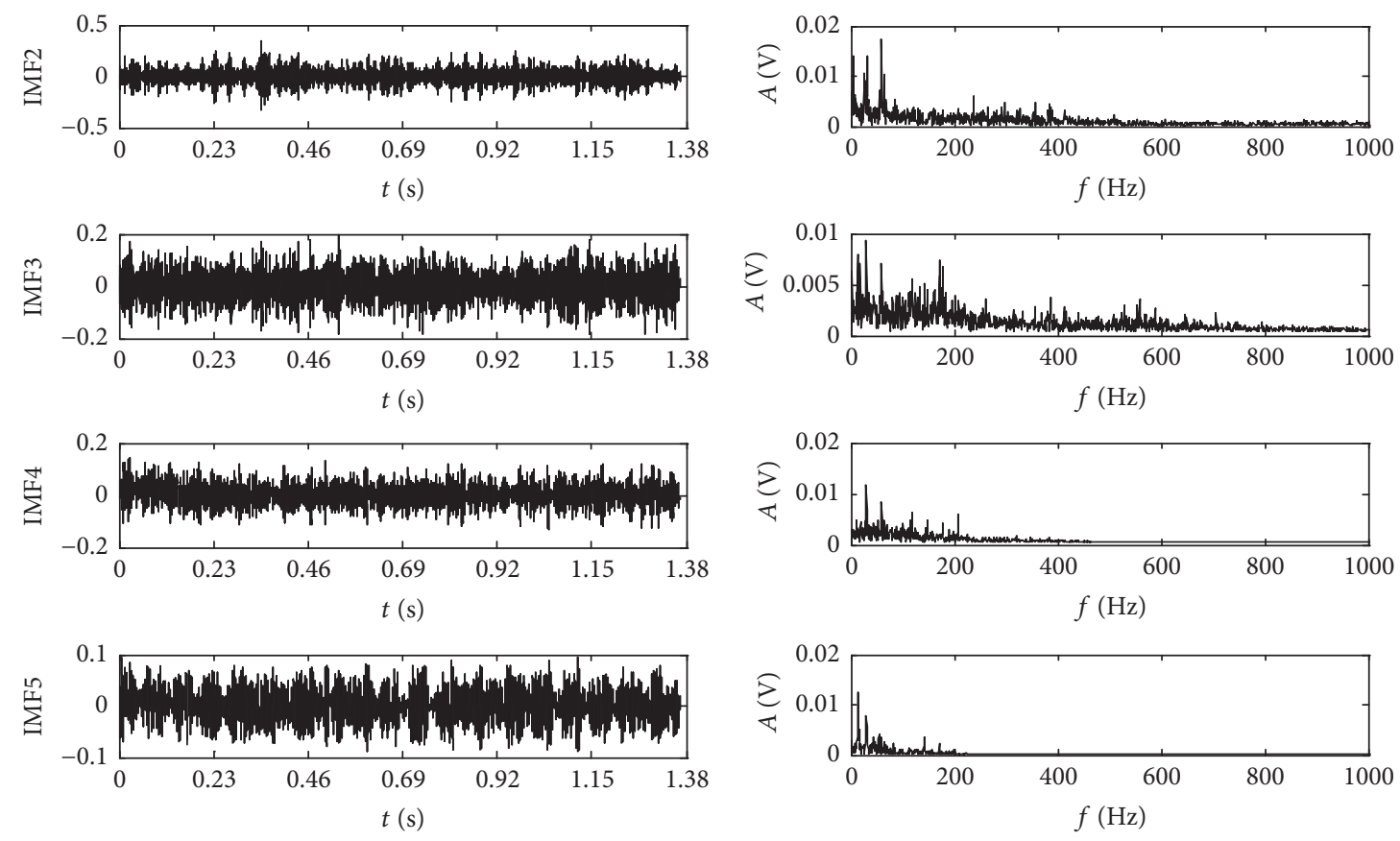

(a)

(b)

FIGURE 8: Analyzed results of EEMD: (a) IMFs; (b) the envelop spectrum of IMFs.

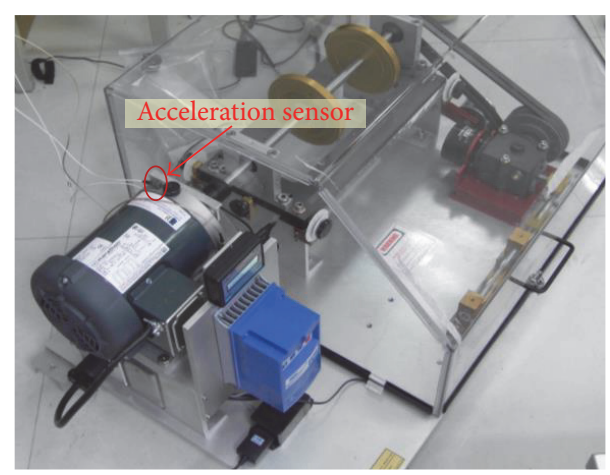

FIGURE 9: SQI's mechanical fault simulator.

Figure 11. Obviously, a lot of background noise is eliminated, and the spectrum peak at $117.2 \mathrm{~Hz}$ is very prominent in the frequency spectrum as shown in Figure 11(b). Therefore, the proposed method in this study can effectively remove useless interferences and enhance the weak feature information submerged in strong noise, thus improving the accuracy of fault identification.

In addition, the method of spectral kurtosis [30] is also adopted to analyze the bearing vibration signal showed in Figure 10(a). The Kurtogram and the resulting signals of spectral kurtosis are illustrated in Figure 12. However, it is difficult to find useful features information relevant to the inner race fault of bearing. Therefore, the two experiments and corresponding comparison results confirm that the proposed method has a better performance for enhancing the weak diagnostic information.
4.3. Engineering Application. With the shortage of fossil fuels and serious environment problems, wind energy sources have drawn intense attention from various countries around the world. However, due to the harsh operation environment, wind turbine, as the important equipment converting wind energy into electrical energy, is often subjected to various loads like wind gust impaction and irregular alternating loads, making some critical components prone to failure. Because of the influence of noisy work environments and strong electromagnetic interference, vibration signals acquired from the generator of wind turbine contain a large amount of noise and interferences to decrease the SNR, which makes it more difficult to detect the fault of bearing at an early stage. Therefore, the proposed method is used for the fault diagnosis of generator bearing in wind turbine and achieves the effective extraction of the feature information from strong background noise.

Through the regular detection of wind turbines in a wind farm, it was found that the vibration response of the front bearing of generator of number 8 wind turbine increased. The installation location of the acceleration transducer is shown in Figure 13. To find out the reasons, the vibration signal needs to be further analyzed. The signals were sampled at $12800 \mathrm{~Hz}$, and the length of data was 16384 . The average rotational speed of the generator was $1406 \mathrm{r} / \mathrm{min}$, and the bearing parameters of generator are displayed in Table 1.

Figure 14(a) shows the original vibration signal of the front bearing of the generator. The inconspicuous impulse components can be found in the waveform of original signal. In Figure 14(b), apart from the rotational frequency of $23.44 \mathrm{~Hz}$, there is not useful feature information relevant 
TABLE 1: Generator bearing parameters of wind turbine.

\begin{tabular}{lcccc}
\hline Type & $\begin{array}{c}\text { Inner diameter } \\
D_{i} / \mathrm{mm}\end{array}$ & $\begin{array}{c}\text { Outer diameter } \\
D_{o} / \mathrm{mm}\end{array}$ & $\begin{array}{c}\text { Roller diameter } \\
d / \mathrm{mm}\end{array}$ & $\begin{array}{c}\text { Number of roller } \\
\text { Contact angle } \\
\beta /\left(^{\circ}\right)\end{array}$ \\
\hline 6324 & 120 & 280 & 41.275 & 8 \\
\hline
\end{tabular}

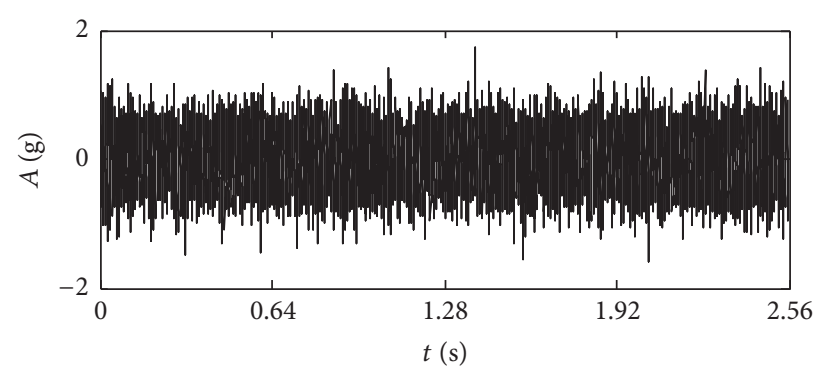

(a)

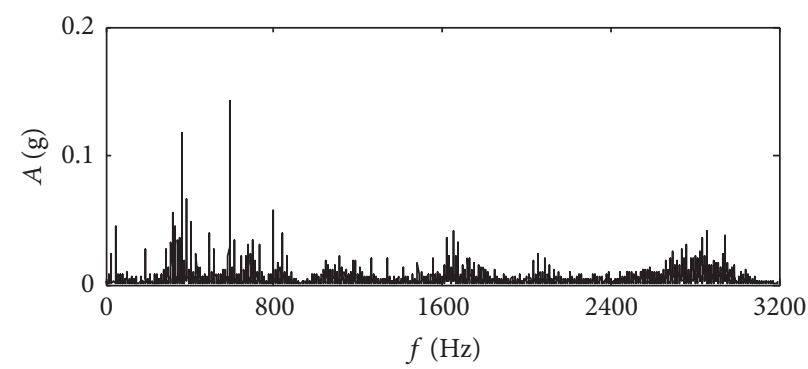

(b)

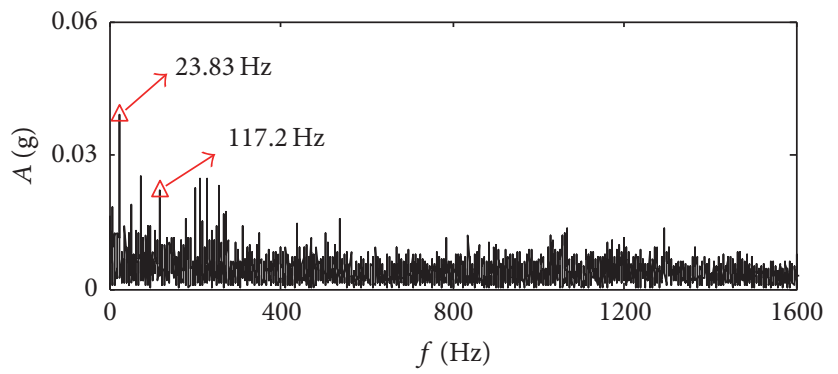

(c)

FIGURE 10: Vibration signal of bearing: (a) the time waveform; (b) the frequency spectrum; (c) the envelop spectrum.

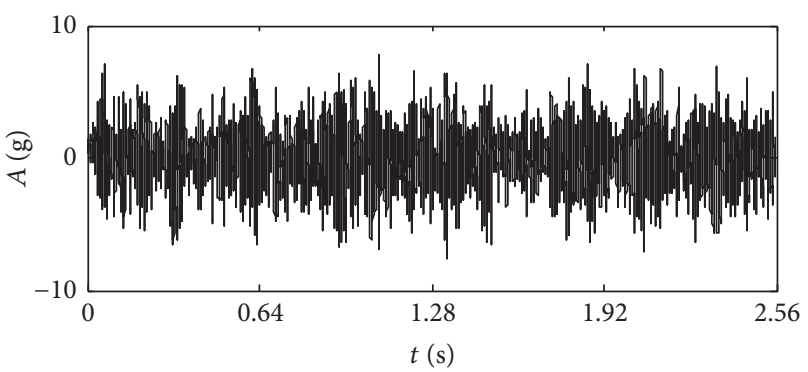

(a)

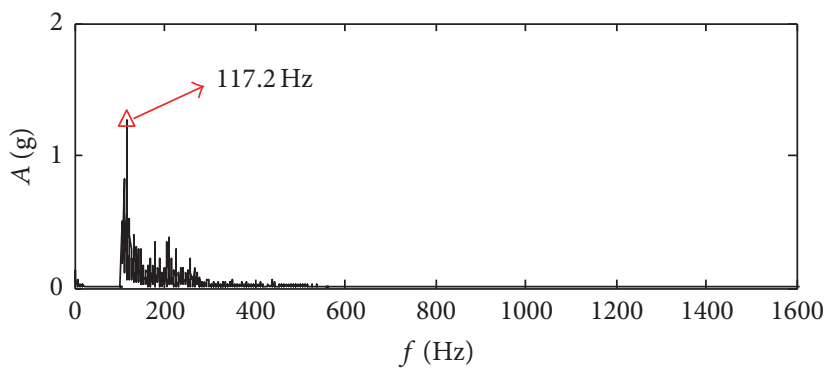

(b)

FIGURE 11: Detection results of the proposed method: (a) the output waveform of SR system; (b) the frequency spectrum.

to bearing fault in the frequency spectrum. And in the Hilbert envelop spectrum, as Figure 14(c) shows, besides the rotational frequency, the spectrum peak at $113.3 \mathrm{~Hz}$ can be found, which consists with the fault characteristic frequency of bearing inner race. However, because of the influence of strong background noise, features of the useful signal are not evident enough to detect faults.

For the purpose of detecting the performance of the generator, the proposed method is applied to process the bearing vibration signal. The algorithm parameters are the same as in the previous section except for the reaching range of noise intensity $\alpha$, which is set to $[0.1,10]$. And the optimal noise intensity obtained by genetic algorithm is $\alpha=6.7$, and the corresponding detection results are displayed in Figure 15. Obviously, a lot of background noise is eliminated, and the spectrum peak at $113.3 \mathrm{~Hz}$ is very highlighted in the spectrum as shown in Figure 15(b), useless interferences are greatly decreased, thereby improving the accuracy of fault identification. The decibel value corresponding to the fault characteristic frequency of bearing inner race calculated by using Shock 


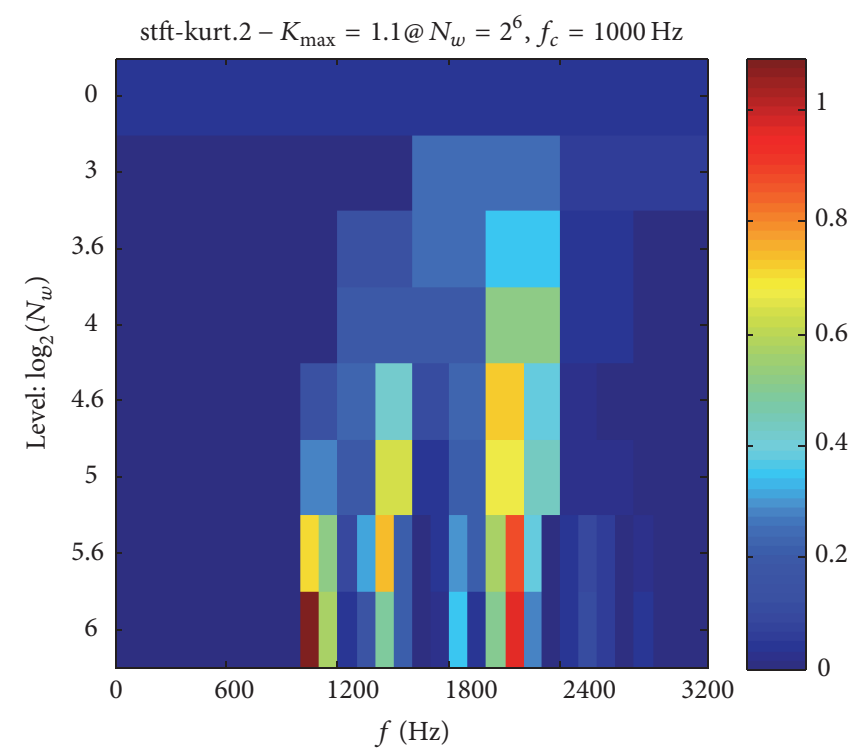

(a)

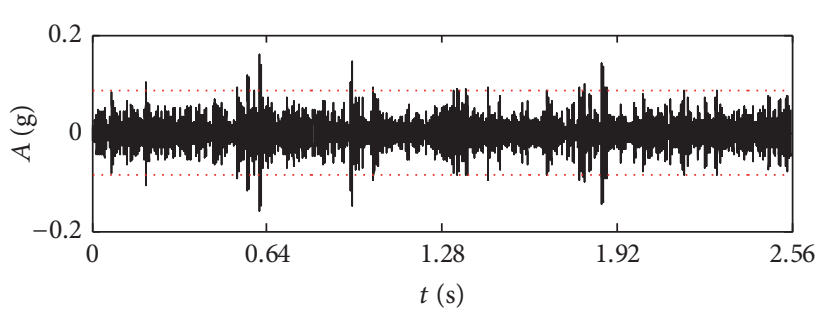

(b)

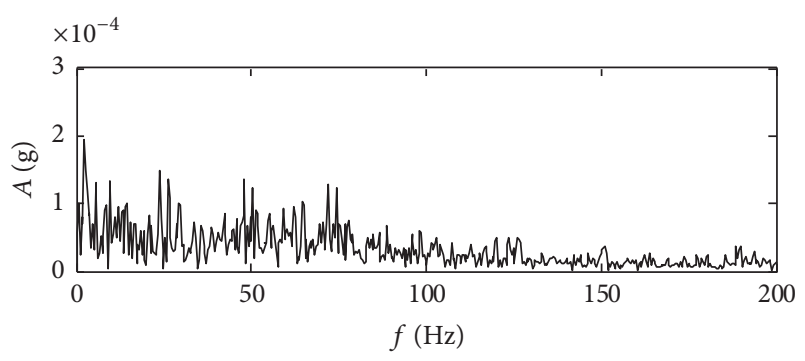

(c)

Figure 12: Analyzed results of spectral kurtosis: (a) the Kurtogram; (b) the purified signal; and (c) the envelop spectrum.

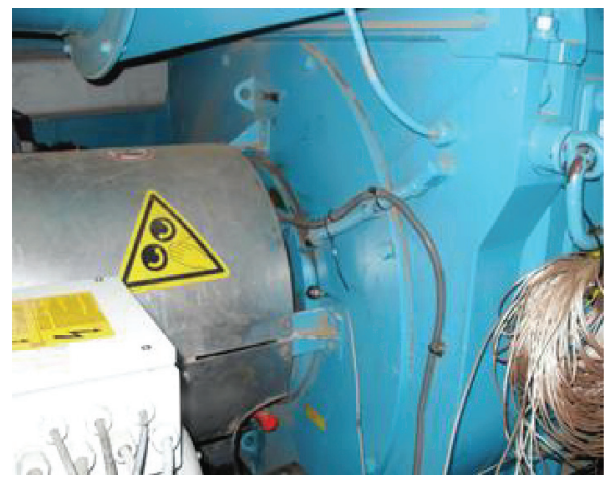

FIGURE 13: The installation location of the acceleration transducer on the front bearing of generator.

Pulse Method is $21.64 \mathrm{~dB}$, which indicates there is an early damage on the bearing inner race. And the bearing of generator should be paid more attention to and detected regularly.

\section{Conclusion}

Aiming at the complexity of vibration signals and the shortcomings existing in multiscale noise tuning based on wavelet decomposition, such as the selection of optimal wavelet bases, a new adaptive multiscale noise control enhanced SR method based on modified EEMD has been investigated in the paper. Compared with wavelet decomposition, EEMD can adaptively decompose the signal into different scales according to the oscillation characteristics of signal itself. And the IMFs at different scales are determined by the signal itself, instead of predetermined kernels, thus strengthening the adaptive capability of the proposed method. And according 


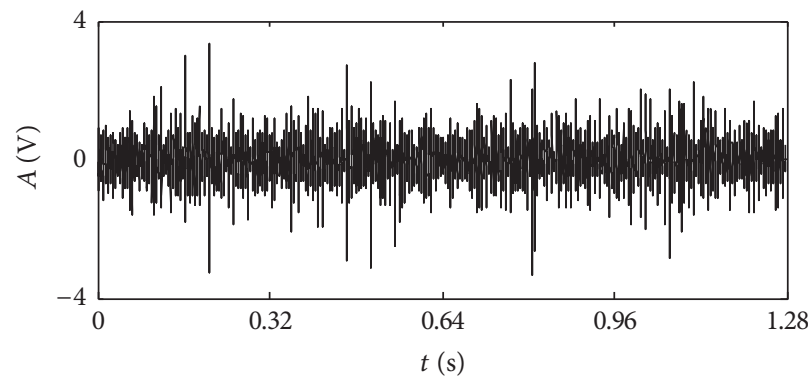

(a)

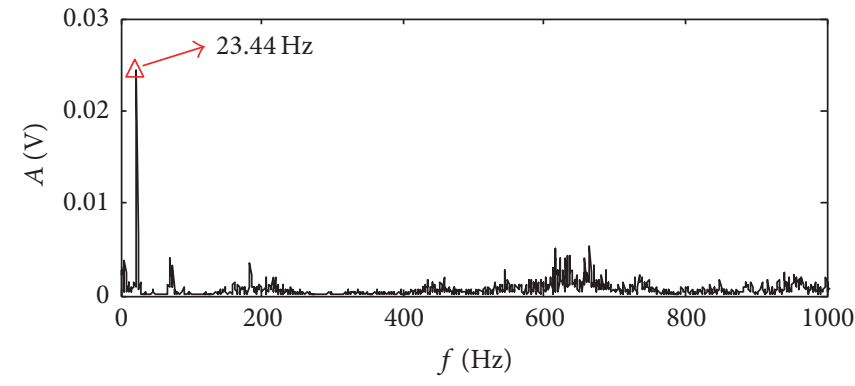

(b)

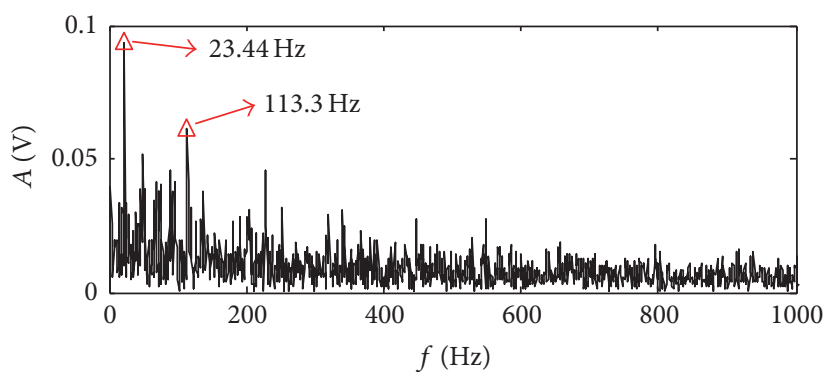

(c)

FIGURE 14: Vibration signal of bearing: (a) the time waveform; (b) the frequency spectrum; (c) the envelop spectrum.

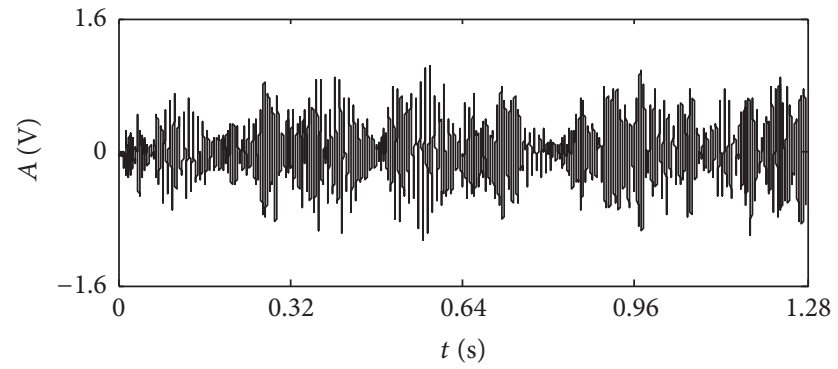

(a)

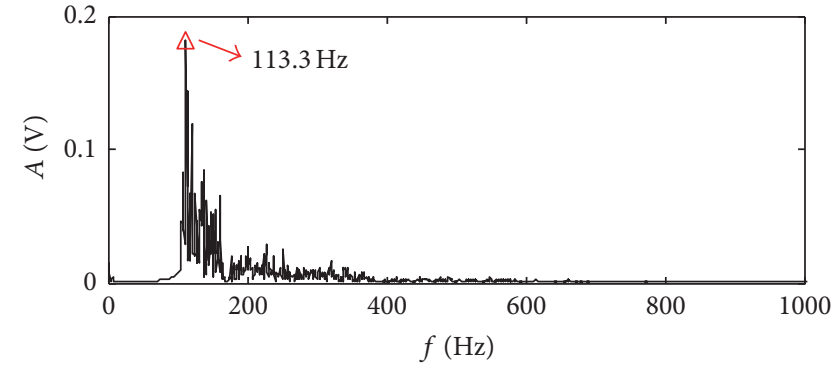

(b)

FIGURE 15: Detection results of the proposed method: (a) the output waveform of SR system; (b) the frequency spectrum.

to the requirements of multiscale noise control algorithm, a modified EEMD algorithm is proposed to effectively reduce the decomposition levels and improve the overall calculation efficiency of the proposed method. By using the algorithm of modified EEMD, not only can the SR be realized by adjusting the noise intensity at different scales with genetic algorithm, but also the orthogonality of adjacent IMFs can be improved to enhance the extraction of weak features from strong noise. Therefore, the adaptive multiscale noise control enhanced SR method based on modified EEMD is conducive to the detection of weak periodic signal in mechanical fault diagnosis. The proposed method has been applied to analyze the bearing vibration signals carrying fault information which are taken from some experiments and wind turbine. The results indicate that the proposed method is able to enhance and extract the fault characteristic information and identify the faults effectively.

\section{Competing Interests}

The authors declare that there are no competing interests regarding the publication of this paper.

\section{Acknowledgments}

This work was supported by the National Natural Science Foundation of China (Grant no. 51505415), China Postdoctoral Science Foundation Funded Project (Grant no. 2015M571279), and Science and Technology Support Project of Qinhuangdao City (Grant no. 201502A008).

\section{References}

[1] X. W. Zhang, R. X. Gao, R. Q. Yan, X. F. Chen, C. Sun, and Z. B. Yang, "Multivariable wavelet finite element-based vibration 
model for quantitative crack identification by using particle swarm optimization," Journal of Sound and Vibration, vol. 375, pp. 200-216, 2016.

[2] R. N. Liu, B. Y. Yang, X. L. Zhang, S. B. Wang, and X. F. Chen, "Time-frequency atoms-driven support vector machine method for bearings incipient fault diagnosis," Mechanical Systems and Signal Processing, vol. 75, no. 15, pp. 345-370, 2016.

[3] Y. K. Akilu, J. K. Sinha, and K. Elbhbah, "An improved data fusion technique for faults diagnosis in rotating machines," Measurement, vol. 58, pp. 27-32, 2015.

[4] Y. Wang, R. Markert, J. Xiang, and W. Zheng, "Research on variational mode decomposition and its application in detecting rub-impact fault of the rotor system," Mechanical Systems and Signal Processing, vol. 60, pp. 243-251, 2015.

[5] K. Dragomiretskiy and D. Zosso, "Variational mode decomposition," IEEE Transactions on Signal Processing, vol. 62, no. 3, pp. 531-544, 2014.

[6] S. Wang, X. Chen, G. Li, X. Li, and Z. He, "Matching demodulation transform with application to feature extraction of rotor rub-impact fault," IEEE Transactions on Instrumentation and Measurement, vol. 63, no. 5, pp. 1372-1383, 2014.

[7] C. Li and M. Liang, "A generalized synchrosqueezing transform for enhancing signal time-frequency representation," Signal Processing, vol. 92, no. 9, pp. 2264-2274, 2012.

[8] G. Cai, X. Chen, and Z. He, "Sparsity-enabled signal decomposition using tunable Q-factor wavelet transform for fault feature extraction of gearbox," Mechanical Systems and Signal Processing, vol. 41, no. 1-2, pp. 34-53, 2013.

[9] J. Yuan, C. J. Wei, B. Zou et al., "A comparative study on multiwavelet construction methods and customized multiwavelet library for mechanical fault detection," Shock and Vibration, vol. 2015, Article ID 963528, 12 pages, 2015.

[10] H. R. Cao, F. Fan, K. Zhou, and Z. J. He, "Wheel-bearing fault diagnosis of trains using empirical wavelet transform," Measurement, vol. 82, pp. 439-449, 2016.

[11] Y. Lei, D. Han, J. Lin, and Z. He, "Planetary gearbox fault diagnosis using an adaptive stochastic resonance method," Mechanical Systems and Signal Processing, vol. 38, no. 1, pp. 113124, 2013.

[12] R. Benzi, A. Sutera, and A. Vulpiani, "The mechanism of stochastic resonance," Journal of Physics A: Mathematical and General, vol. 14, no. 11, pp. L453-L457, 1981.

[13] C. Nicolis and G. Nicolis, "Stochastic aspects of climatic transitions-additive fluctuations," Tellus, vol. 33, no. 3, pp. 225234, 1981.

[14] R. Zhao, R. Q. Yan, and R. X. Gao, "Dual-scale cascaded adaptive stochastic resonance for rotary machine health monitoring," Journal of Manufacturing Systems, vol. 32, no. 4, pp. 529535, 2013.

[15] X. F. Zhang, N. Q. Hu, L. Hu, and Z. Cheng, "Multi-scale bistable stochastic resonance array: a novel weak signal detection method and application in machine fault diagnosis," Science China Technological Sciences, vol. 56, no. 9, pp. 2115-2123, 2013.

[16] Y. Qin, Y. Tao, Y. He, and B. P. Tang, "Adaptive bistable stochastic resonance and its application in mechanical fault feature extraction," Journal of Sound and Vibration, vol. 333, no. 26, pp. 7386-7400, 2014.

[17] D. Y. Han, P. Li, S. J. An, and P. M. Shi, "Multi-frequency weak signal detection based on wavelet transform and parameter compensation band-pass multi-stable stochastic resonance," Mechanical Systems and Signal Processing, vol. 70-71, pp. 9951010, 2016.
[18] J. Wang, Q. B. He, and F. R. Kong, "Adaptive multiscale noise tuning stochastic resonance for health diagnosis of rolling element bearings," IEEE Transactions on Instrumentation and Measurement, vol. 64, no. 2, pp. 564-577, 2015.

[19] X.-H. Chen, G. Cheng, X.-L. Shan, X. Hu, Q. Guo, and H.-G. Liu, "Research of weak fault feature information extraction of planetary gear based on ensemble empirical mode decomposition and adaptive stochastic resonance," Measurement, vol. 73, pp. 55-67, 2015.

[20] Z.-H. Lai and Y.-G. Leng, "Generalized parameter-adjusted stochastic resonance of duffing oscillator and its application to weak-signal detection," Sensors, vol. 15, no. 9, pp. 21327-21349, 2015.

[21] J. Li, X. Chen, Z. Du, Z. Fang, and Z. He, "A new noise-controlled second-order enhanced stochastic resonance method with its application in wind turbine drivetrain fault diagnosis," Renewable Energy, vol. 60, pp. 7-19, 2013.

[22] F. B. Duan, F. Chapeau-Blondeau, and D. Abbott, "Doublemaximum enhancement of signal-to-noise ratio gain via stochastic resonance and vibrational resonance," Physical Review E, vol. 90, no. 2, Article ID 022134, 2014.

[23] N. E. Huang, Z. Shen, S. R. Long et al., "The empirical mode decomposition and the hilbert spectrum for nonlinear and nonstationary time series analysis," Proceedings of the Royal Society of London Series A: Mathematical, Physical and Engineering Sciences, vol. 454, no. 1971, pp. 903-995, 1998.

[24] Z. Wu and N. E. Huang, "Ensemble empirical mode decomposition: a noise-assisted data analysis method," Advances in Adaptive Data Analysis, vol. 1, no. 1, pp. 1-41, 2009.

[25] Y. Lei, Z. He, and Y. Zi, "Application of the EEMD method to rotor fault diagnosis of rotating machinery," Mechanical Systems and Signal Processing, vol. 23, no. 4, pp. 1327-1338, 2009.

[26] Q. He, J. Wang, Y. Liu, D. Dai, and F. Kong, "Multiscale noise tuning of stochastic resonance for enhanced fault diagnosis in rotating machines," Mechanical Systems and Signal Processing, vol. 28, pp. 443-457, 2012.

[27] B. Hu and B. Li, "A new multiscale noise tuning stochastic resonance for enhanced fault diagnosis in wind turbine drivetrains," Measurement Science and Technology, vol. 27, no. 2, Article ID 025017, 2016.

[28] J. J. Zhang and T. Zhang, "Parameter-induced stochastic resonance based on spectral entropy and its application to weak signal detection," Review of Scientific Instruments, vol. 86, no. 2, Article ID 025005, pp. 1-6, 2015.

[29] http://csegroups.case.edu/bearingdatacenter/home.

[30] Y. X. Wang, J. W. Xiang, R. Markert, and M. Liang, "Spectral kurtosis for fault detection, diagnosis and prognostics of rotating machines: a review with applications," Mechanical Systems and Signal Processing, vol. 66-67, pp. 679-698, 2016. 


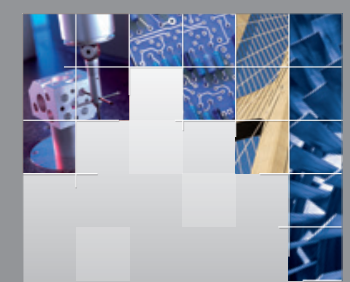

\section{Enfincering}
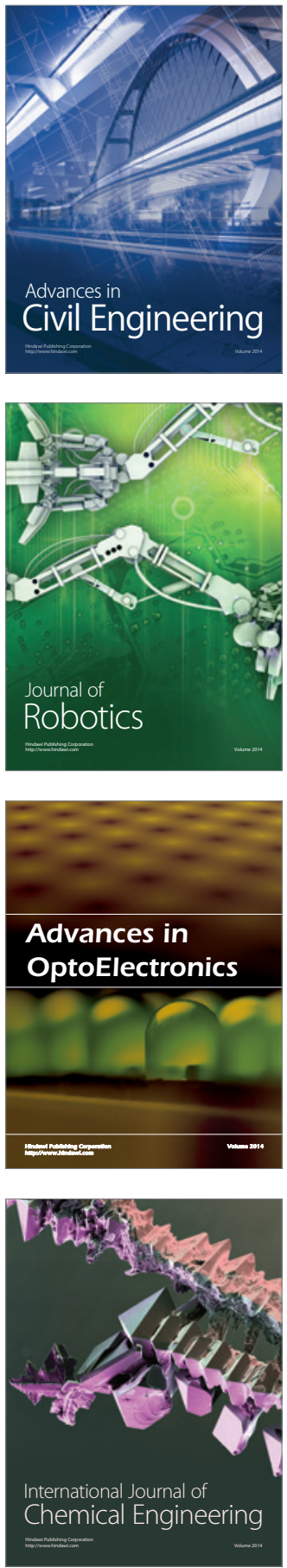

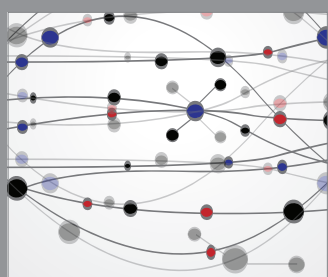

The Scientific World Journal

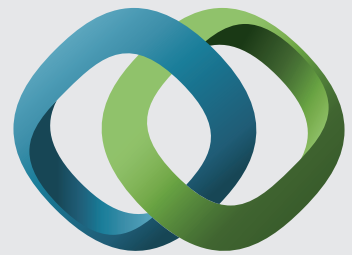

\section{Hindawi}

Submit your manuscripts at

http://www.hindawi.com
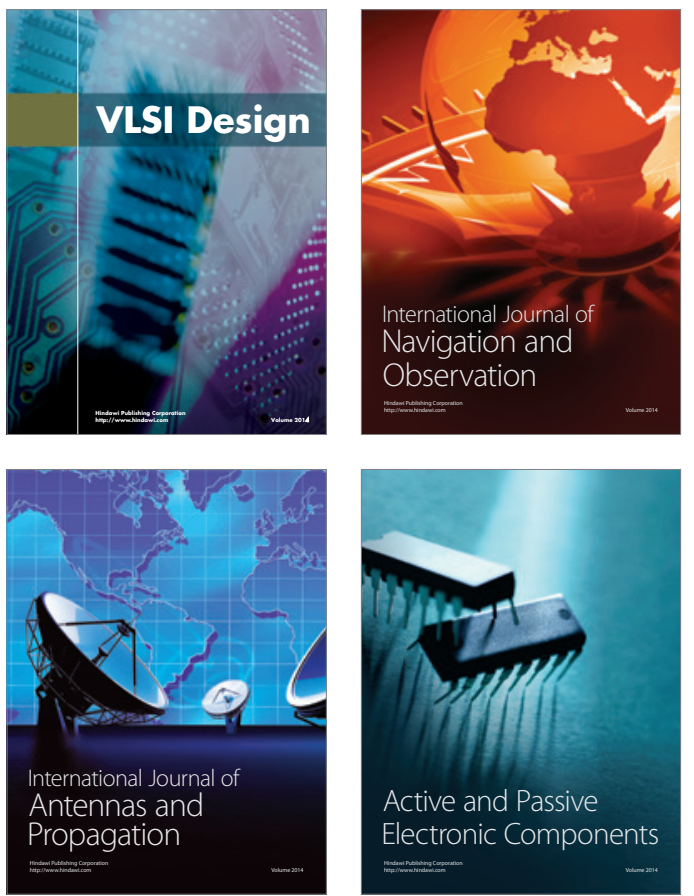
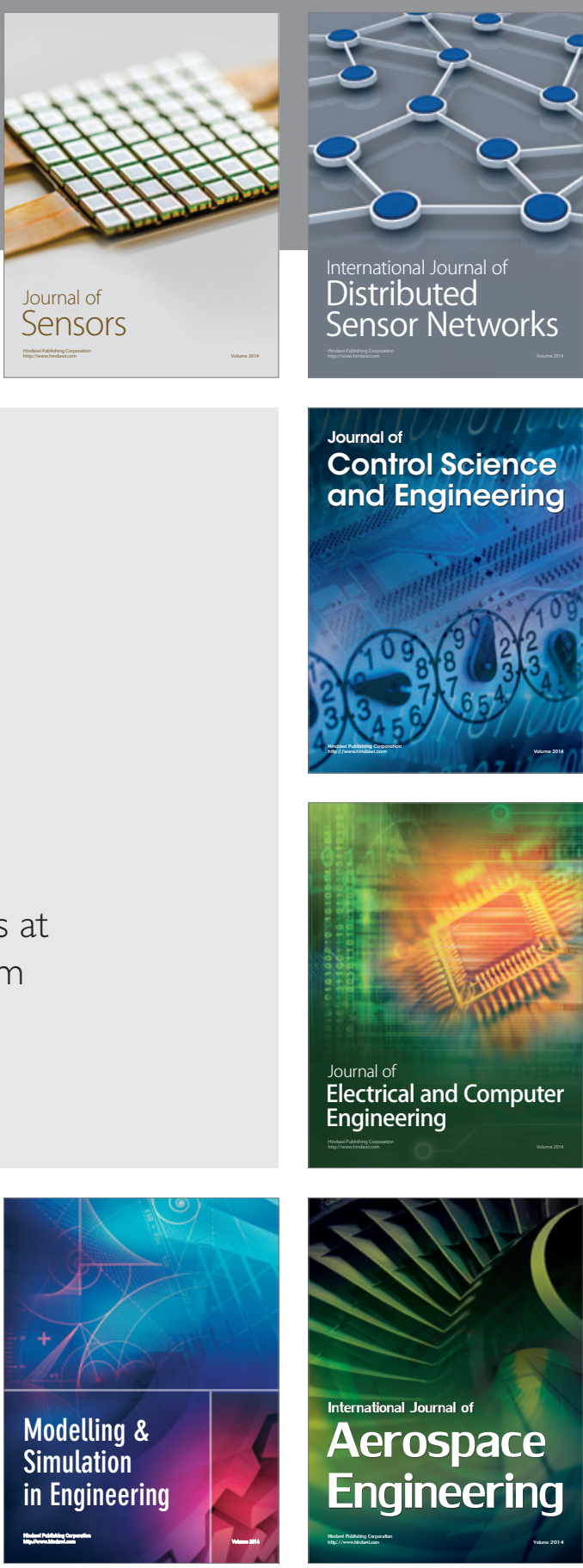

International Journal of

Distributed

Sensor Networks

Journal of

Control Science

and Engineering
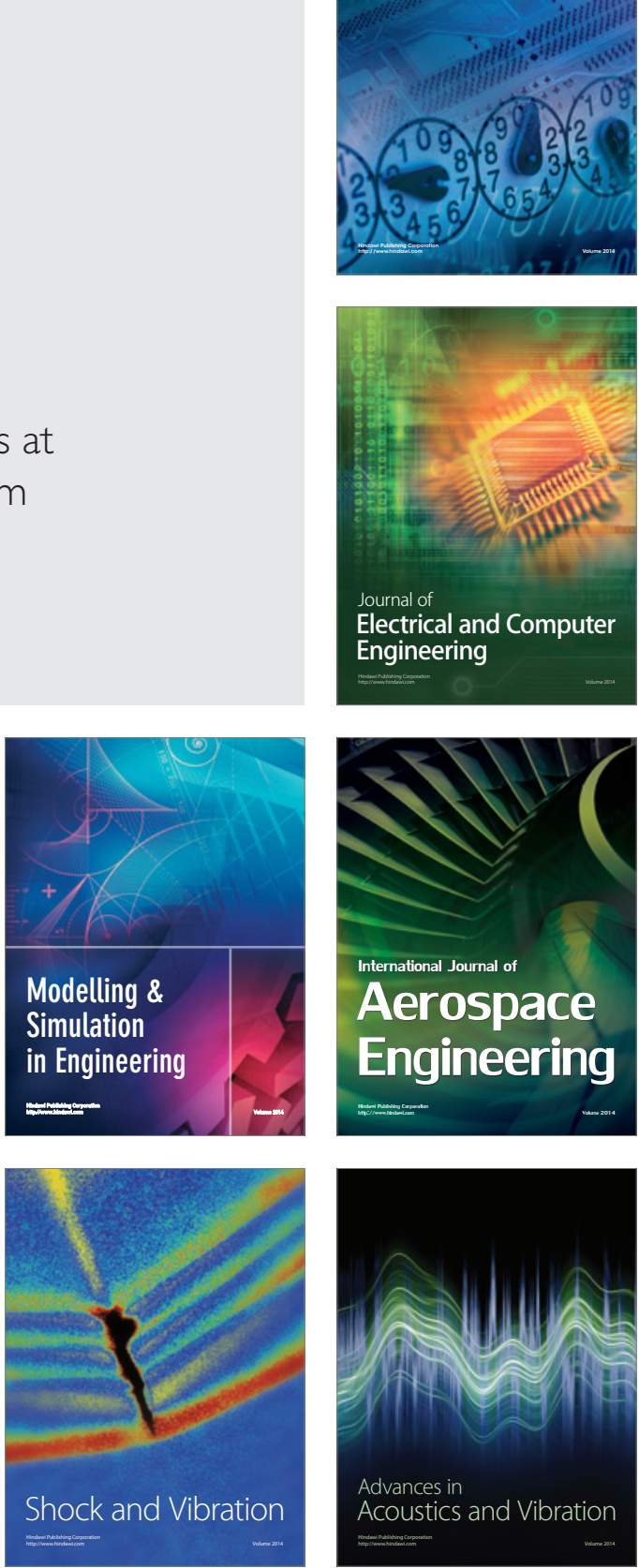